\title{
Posibilidades de investigación sobre instituciones administrativas, económicas y sociales de la edad contemporánea en el Archivo General de la Administración
}

\author{
José Francisco Fornies Casals \\ (Universidad de Alcalá de Henares)
}

El Archivo General de la Administración a los efectos de la realización de investigaciones históricas relacionadas con las instituciones administrativas, sociales y económicas de nuestro país es un centro de primordial importancia, dada la gran cantidad de documentación que conserva procedente fundamentalmente de los restantes archivos de organismos oficiales. De ahí, que me haya planteado el hacer un análisis de las posibilidades que presenta en esta línea argumental, para hacer una invitación permanente a investigadores, tanto de la Universidad de Alcalá de Henares como de otras Universidades, a que utilicen unos conjuntos documentales absolutamente vitales para el conocimiento de nuestra historia contemporánea.

En efecto, los descriptores consultados sobre los contenidos del Archivo, nos ponen en la pista de la documentación que procede de los Ministerios de Agricultura, Asuntos Exteriores, Comercio, Cultura, Educación, Hacienda, Industria, Información y Turismo, Interior, Justicia, Obras Publicas, Presidencia de Gobierno, Secretaría General del Movimiento, Sección Femenina y Trabajo; de todos estos he segregado un grupo extenso de temas de investigación, relacionados con las instituciones administrativas, sociales y económicas, susceptibles de investigaciones en conjunto, o equipo, o de realizar tesis doctorales, tesinas, y artículos científicos, que por Ministerios y organismos emisores son los que a continuación describo.

En la sección de Agricultura se conserva documentación institucional sobre la Agencia Nacional del Tabaco, las almadrabas y el Instituto Nacional de Investigaciones Agronómicas, suficiente como para poder 
hacer estudios completos de los mismos, en el caso de los dos últimos con nivel de tesis. Otras series de interés son las concernientes a bosques (1911-1969), estadisticas agrarias (1935-1950), fomento e importación ganadera (1903-1971), o la ordenación de cultivos en España entre 1912 y 1970. En Asuntos Exteriores la documentación versa sobre la correspondencia de nuestras legaciones diplomáticas en 15 países, desde los E.E.U.U. hasta la Santa Sede, y con fechas que se remontan en ocasiones, al siglo XVI, siendo muy interesante lo perteneciente al funcionamiento propiamente dicho de las citadas embajadas.

En la sección del Ministerio de Cultura, desde el prisma institucional del propio Ministerio, se conserva la documentación relativa a sus inmuebles, obras, correspondencia y administración, desde 1938 a 1981, en tanto que los fondos sobre las bibliotecas dependientes de la Dirección General del Libro, comprenden los años de 1928 hasta 1982. Igualmente se conserva toda la documentación del Centro Nacional de Información Artística, que contaba con archivo propio, y que se comenzó a formar tras la guerra civil, con fondos relacionados con el Patrimonio Artístico y su situación durante la contienda ${ }^{1}$. Otras series interesantes de la sección, son las procedentes de excavaciones arqueológicas, entre 1962 y 1981, y la de teatros nacionales entre 1958 y 1976.

En la documentación sobre el Ministerio de Comercio destaca la serie del Instituto de Moneda Extranjera, desde 1951 a 1974, es decir todo el período de creación y funciones en época de Franco. Los 1.415 legajos relativos a la construcción naval entre 1939 y 1970, constituyen otra serie destacable, al contener los datos sobre botaduras, construcción naval por empresas, crédito naval y registro general, es decir lo más trascendente sobre la evolución de nuestra marina mercante, asi como sobre las escuelas y enseñanzas marítimas, desde la formación de patrones, hasta la de mecánicos navales. Dentro de esta sección de Comercio, conviene reseñar la existencia de dos subsecciones de evidente interés, una la concerniente a la ayuda americana ${ }^{2}$, desde 1952 a 1977 , y otra, la que versa acerca de las inversiones españolas en el extranjero desde 1965 a 1974.

La sección de Educación es una de las más abundantes en posibilidades para la historia institucional contemporánea, en la misma se hallan los documentos básicos para el estudio de los archivos en España entre

Sobre el contenido del citado archivo, véase: L. Alvarez Lopera, La Politica de bienes culturares del gobierno republicano, Madrid, 1982.

2 El tema fue estudiado en sus principales aspectos por A. Viñas, Los Pactos secretos de Franco con los E.E.U.U., bases, ayuda económica, recortes de soberania, Barcelona, 1977. 
1936 y 1944, la Calcografía Nacional, el Consejo de Instrucción Pública desde 1855 a 1930, las Escuelas de Artes y Oficios desde 1924 a 1964, el Instituto Jorge Juan desde 1915 a 1980, el propio Ministerio de Instrucción Pública desde 1831 hasta 1908, la Junta de Ampliación de Estudios de 1907 a 1938, el Patronato Juan de la Cierva de 1932 a 1979, y las Sociedades Artísticas y Culturales desde 1940 a 1969. De evidente importancia son además las series documentales relativas a las excavaciones arqueológicas entre 1842 y 1934, asi como los expedientes de las exposiciones nacionales desde 1922 a 1970.

Las instituciones identificables en los fondos procedentes del Ministerio de Hacienda susceptibles de estudio son el Banco de Crédito Agrícola desde su fundación hasta 1975, el Montepio Civil del Estado y el Montepío Militar, cuyos libros de consignaciones abarcan los años de 1900 hasta 1969, si bien el conjunto documental más importante lo constituye el relativo al sector seguros, que se remonta a 1899 y llega a 1963, y que una vez analizado, puede dar las claves básicas para el estudio del mismo en nuestro país, dentro de las distintas modalidades que lo han caracterizado, es decir riesgos diversos, de transporte, de vida, de incendios, obligatorio de vehículos, de aviación, de carretera, de ferrocarril, marítimos y sanitarios. Como series destacables del propio Ministerio, pueden citarse las relativas a las contribuciones, remontándose la matrícula industrial desde 1856 hasta 1940, la rústica de 1900 a 1923, la urbana de Madrid de 1905 a 1952, y los padrones de la capital entre 1904 y 1973. Igualmente se hallan en la sección los 20 volúmenes del registro de entrada de bienes nacionales entre 1835 y 1859 procedentes de desamortización, y documentación variada sobre la deuda pública entre 1814 y $1931^{3}$.

También el fondo archivistico del Ministerio de Industria presenta posibilidades de investigación histórica institucional, pues cuenta con toda la documentación fundacional a partir de 1929 y su evolución posterior hasta 1969, de la Compañía Española de Petróleos Cepsa. Igualmente se custodia la documentación de la Empresa Nacional Calvo Sotelo desde su

3 Tanto el tema de la desamortización, como el de la deuda pública, están de plena actualidad historiográfica. Tras las obras globales de F. TOMÁS y VALIENTE, y de F. SIMÓN SEGURA, han ido apareciendo monografias concretas referentes a lo procesos pormenorizados de desamortización en provincias, como es el caso de la vallisoletana, debida a G. RuEDA. Los estudios sobre la deuda pública y su incidencia en el desenvolvimiento de la Hacienda desde el pasado siglo, fueron objeto de la atención de J.M. TalladA PAULi, y de J. SARDa DeXeus, mas hoy, gracias a los trabajos de F. Comin, Hacienda y economia en la España contemporánea (1800-1936), Madrid, 1988, y de A. Carreras (coordinador), Estadísticas históricas de España. Siglos XIX y XX, Madrid, 1989, esta información es mucho más exhaustiva. 
creación en 1942 hasta 1975, así como los libros del Consejo de Administración de la Compañía de Minas de Almadén desde 1929 a $1970^{4}$. De indudable interés son también las series del Consejo de Economía Nacional que abarcan a los años 1920-1930, y los 320 legajos de la Dirección General de Pequeña y Mediana Empresa, cuyo análisis permitiría conocer a fondo la importancia de este sector económico tan fomentado por el régimen franquista, y que arranca de 1963 para llegar hasta $1982^{5}$.

La estructuración y el funcionamiento institucional del Ministerio de Información y Turismo entre 1952 y 1976 , puede ser analizado ampliamente al conservarse un fondo legislativo completo sobre el mismo y toda la documentación de la inspección de servicios. Además está en el Archivo la mayor parte de la documentación generada por la Escuela Oficial de Periodismo entre 1942 y 1976 , que puede dar lugar a un estudio monográfico, sobre el sector durante el franquismo. En relación con la figura del propio Francisco Franco se conservan en esta sección unos legajos de correspondencia que abarcan los años de 1965 a $1973^{6}$; y también como curiosidad, hay 24 legajos, bajo el epigrafe Gibraltar, donde se han recopilado informaciones gráficas relativas a nuestro contencioso, en los años en que estuvo activo este Ministerio.

Del Ministerio de la Gobernación, hoy de Interior, se custodian los documentos de todas las asociaciones dependientes de la Dirección General de Política Interior, entre 1941 y 1963, así como de las acogidas al régimen jurídico del Movimiento, entre 1958 y 1977, con una amplitud de fondos suficientes para un estudio global de las tendencias políticas dentro de la legalidad. En paralelo, se pueden analizar también las asociaciones y actividades políticas y sindicales ilegales, pues se conservan los expedientes abiertos por la Guardia Civil entre 1940 y 1977, en un amplio fondo compuesto de 555 legajos. Otra de las instituciones cuya documentación se conserva prácticamente íntegra es la de la Caja de Depósitos, desde 1852 hasta 1879 , que si bien ha sido objeto de un estudio amplio ${ }^{7}$, sigue siendo muy útil, para el análisis de la evolución de nuestro sistema financiero en la centuria pasada. En esta sección de Interior se conservan además series

\footnotetext{
4 La historia de estas minas centenarias, ha sido abordada últimamente por V. MARTin MARTIN, Los Rothschild y las minas de Almaden, Madrid, 1980.

5 En relación con la problemática suscitada por estas empresas a la hora de su implantación, véase: C. Hornillos, Problemas de la pequeña y mediana industria en España, Madrid, 1969.

6 No estaría de más hacer un cotejo de esta información con la vertida en las obras de $F$ Franco Salgado-Araujo, R. de la Cierva, y J.P. Fusi, dedicadas al personaje.

7 Véase: a L. Gonzalo y GonzAlez, El tesoro público y la Caja General de Depositos 1852. 1868, Madrid, 1.981
} 
documentales sobre beneficiencia, que son complementarias a las existentes en la sección propia, y que atañen al personal médico y sanitario, a los perceptores de la Cruz de Beneficiencia, y al funcionamiento de varios hospitales desde 1896 hasta 1918. Por último, existe también documentación variada sobre instituciones muy poco estudiadas como son las casas de préstamos, cuya decadencia desde comienzos del siglo XX, era notoria, pero que habian jugado un papel relevante a nivel de economia local durante la segunda mitad del siglo XIX.

La documentación del Ministerio de Justicia permitirá el análisis de diversas instituciones, de amplia repercusión entre la ciudadanía. En primer lugar, el Servicio Jurídico del Estado, adscrito a la Dirección General del mismo nombre, puede ser estudiado globalmente, pues la serie documental abarca desde 1929 hasta 1975. Lo mismo puede decirse de varios tribunales con competencias especificas; así ocurre con el Tribunal de Responsabilidades políticas, cuyos 209 legajos, abarcan los años de 1939 a 1946; a su vez el Tribunal de Orden Público está representado por 1.484 legajos, de los años comprendidos entre 1964 y 1975. De más entidad, el Tribunal Supremo, en su sala de lo civil, está representado por documentación desde 1904 a 1952, dividida en apartados especificos, con un total de 1.246 legajos. La Audiencia Territorial de lo Penal de la Provincia de Madrid entre 1894 y 1960 puede ser analizados juzgado por juzgado, de los 21 que la componian, desde un enfoque institucional, o por aspectos globales como la tipología del delito, la delincuencia por grupos sociales, la frecuencia delictiva, etc. A su vez los delitos monetarios entre 1939 y 1976, los delitos menores entre 1930 y 1973, y las acciones tomadas contra vagos y maleantes en los Juzgados de Instrucción y en la Audiencia Nacional entre 1945 y 1966, pueden ser objeto de trabajos específicos, dadas las series documentales existentes.

En cuanto a instituciones complementarias de la acción de la justicia, el Archivo custodia los fondos completos de la Dirección General de Instituciones Penitenciarias desde 1938 hasta 1975, lo que puede propiciar estudios acerca de la organización interior de los recintos penitenciarios, al guardarse los expedientes de contabilidad, obras, y expedientes de funcionarios, así como el análisis de la ejecución de la política penitenciaria, pues están los expedientes relativos a libertad condicional, asignaciones familiares, reducción de penas por el trabajo, invalidaciones y recompensas. Igualmente pueden analizarse los centros destinados a la protección del menor entre 1925 y 1971, así como los expedientes relativos a la reparación de templos entre 1939 y 1971, emprendida desde la Dirección General de Asuntos Religiosos. 
Procedente del Ministerio de Obras Públicas hay una amplia gama de series documentales entre las que destaca la concerniente a los transportes. En primer lugar el Consejo Superior de Transportes Terrestres está ampliamente documentado desde 1909 a 1949; después hay copiosa documentación sobre ferrocarriles desde 1844 a 1975, desde las primeras concesiones, proyectos, etc., hasta lo relacionado con las obligaciones hipotecarias, es decir los aspectos más modernos de financiación ${ }^{8}$. La serie de legajos sobre los tranvías en España entre 1870 y 1970 es también muy completa, así como la concerniente a las concesiones de líneas de autobuses entre 1920 y 1940 . Con respecto a las obras públicas propiamente dichas, los legajos sobre puertos son copiosísimos, ordenados por provincias y abarcando desde 1848 a 1965. Las obras hidráulicas se remontan en la documentación hasta 1852, y llegan a 1967, y en la misma se recogen los proyectos y realizaciones de canales y presas; en esta línea argumental el Canal de Isabel II está representado desde 1833 a 1964, los planes de riego de 1816 a 1971, en tanto que la Confederación Hidrográfica del Ebro, puede ser analizada desde 1902 hasta los años 40, división por división. En el apartado de previsión dependiente de este Ministerio, se hallaban la Caja Ferroviaria y el Montepío de Puertos, cuya documentación está recogida en su mayor parte en el Archivo. Por último, de este Ministerio se conserva un amplio fondo sobre proyectos de edificios religiosos de 1939 a 1975, así como las series correspondientes a la extinta Dirección General de Regiones Devastadas, desde 1938 a 1961.

Del Ministerio de la Presidencia del Gobierno se conservan series documentales en sumo copiosas, desde un enfoque institucional. En primer lugar, se custodia un amplio material procedente del Consejo de Estado, que abarca desde 1831 hasta 1957; sobre el Consejo de Ministros, los libros de actas, avanzan desde 1824 a 1873 en lo concerniente a sesiones, en tanto que en acuerdos, las series van de 1854 a 1873 y de 1907 a 1931. Sobre la organización de los cuerpos legisladores hay una buena serie documental desde 1822 a 1957, pero donde realmente los fondos son abundantes y muy completos, es en los asuntos de Africa. Sobre el Protectorado de Marruecos hay 3.194 legajos, que abarcan la gobernación, política, ejército, beneficencia, educación, personal administrativo, justicia, hacienda, transportes, bienes y rentas, sanidad, administración local, obras públicas y comunicaciones. Después, con una subdivisión similar, los fondos sobre los gobiernos de Guinea, Ifni, Sahara Español,

8 Información esta que completaría la aportada en los trabajos de A. CASARES ALONSO, F. WAIS, y la coordinada por M. ARTOLA en la obra colectiva editada en 1978. 
Ceuta y Melilla, se remontan de los comienzos hasta la supresión de los mismos, e incluso a los antecedentes del siglo XVIII, como ocurre en Marruecos, y las plazas de Ceuta y Melilla ${ }^{9}$. Instituciones de menor fuste, con series documentales suficientes para su estudio, también dependientes del Ministerio de Presidencia del Gobierno, son el Colegio de Huérfanos de Guerra, de 1876 a 1917, el Comité Sindical del Cacao, de 1937 a 1974, y la Delegación Peninsular para el Café de las Cámaras Agrícolas de Guinea, de 1944 a 1976.

Del Ministerio de Trabajo, el Archivo contiene algunas series documentales sobre instituciones representativas y susceptibles de estudios monográficos, como es el caso de la Magistratura del Trabajo entre los años de 1939 a 1975, en sus tres salas de actuación. El Iristituto de Mediación, Arbitraje y Conciliación, I.M.A.C., entre 1962 y 1977, es otro ejemplo de Institución cuya intervención en las relaciones laborales en el periodo franquista fue muy destacada; además la serie de 1.944 legajos referentes al cooperativismo en España de 1944 a 1977, también puede constituir un estudio sobre esta fórmula de organización de la producción, y sobre las instituciones a que dio origen. Como colofón a esta descripción básica de las series documentales existentes en el Archivo General de la Administración, que pueden dar origen a investigaciones institucionales puntuales, sólo me queda por citar la existencia de dos series, que darán la çlave de la evolución de dos instituciones netamente franquistas, una la Secretaría General del Movimiento, y otra la Sección Femenina, desde 1940 a 1975, dado que guardan todos los legajos generados por ambas.

A la vista de cuanto he expuesto hasta aquí, parece lógico que ahora haga algunas reflexiones, unas de tipo descriptivo, y otras de tipo epistemológico. En primer lugar, ha de hacerse referencia a los espacios cronológicos que aparecen mejor representados, ya que estos son la época de la Restauración, de la Dictadura de Primo de Rivera hasta la Guerra Civil, y por último, y con una extensión muy superior, el espacio temporal que denominamos hoy por hoy franquismo, con una simplicidad de concepto definitorio, que tal vez vaya siendo hora de empezar a variar.

Entrando en una segunda reflexión, es preciso tener en cuenta que la realización de juicios de valor sobre los acontecimientos pretéritos en materia económica, social, cultural y política, sin admitir las limitaciones impuestas

9 Del interés que suscita el análisis de nuestro colonialismo conternporáneo son buenas muestras los trabajos de $V$. MORALES LEzCANo, si bien es cierto que aún queda mucho por conocer, y que la complejidad de la documentación conservada puede reservar muchas sorpresas. 
por las posibilidades de acción de que dispusieron en cada momento los actores de la historia, nos puede conducir a confeccionar una historia incomprensible, radicalizada y cargada de apreciaciones injustas.

Hechas ambas reflexiones aclaratorias, se impone analizar cuales fueron las posibilidades de acción de que dispusieron los españoles entre 1939 y 1975 para influir o cambiar el devenir económico, social, cultural y político de su nación. La historiografía del período franquista está a mi juicio enormemente sesgada entre detractores y panegiristas, porque viene mediatizada por la negación o la aceptación de los resultados de la Guerra Civil, o lo que es lo mismo, la contienda citada, en su vertiente ideológica, aún conserva frentes activos, es más, creo que no hay una voluntad clara entre los historiadores españoles, asi como entre los hispanistas que han analizado nuestra historia reciente, de sentarse a firmar la paz definitiva, lo que conllevaría a enfocar esta historia de manera muy diferente, y a renunciar a muchos de sus postulados estereotipados.

En consecuencia, deseo apostar por el futuro de esta proposición, que pasaría por la aceptación, como hechos ya consumados, de los límites permanentes que se le impusieron a la nación en materia económica, social, cultural y política, y a partir de ahí, profundizar en el conocimiento de una generación, la que intencionalmente podría llamarse, la generación sacrificada, que correspondería a quienes se hallaban entre los 15 y los 30 años en 1939, al objeto de ver cuales fueron sus pretensiones, sus fracasos o sus logros, y hasta que punto fueron capaces de superar esas limitaciones.

Mi propuesta, en concordancia con cuanto he sugerido, sería la de retomar la historia del pueblo español durante el franquismo, para darle el protagonismo que hasta ahora se le ha negado, ante la abrumadora atención que el General Franco, y los aspectos formales limitantes de su régimen han recibido, tanto de quienes lo denostaron y denuestan, como de quienes 10 aplaudieron y aplauden. Este nuevo planteamiento tendría como objetivo primordial el hacer recobrar al pueblo español el papel auténtico de su dimensión, empezando por considerar a Francisco Franco y a su estructura dictatorial condicionante, más como una anécdota coercitiva, que como el factor axial del período.

Teniendo en cuenta estos planteamientos básicos, esta comunicación va destinada también a valorar las posibilidades de investigación que brinda el Archivo General de la Administración, donde entre la compleja variedad de sus fondos institucionales, y a pesar de la rigurosidad formal aparente de sus conjuntos documentales, procedentes como apuntaba anteriormente de organismos oficiales, subyace además una información valiosisima para alcanzar en parte esa meta historiográfica apuntada, de 
devolver al pueblo español el protagonismo de la historia reciente de nuestra nación, que va de 1939 a 1975. Detrás, o en el interior incluso de cada expediente, hay seres humanos, que lo realizaron, y seres humanos que intervinieron en todo cuanto se trata o acontece. Busquemos ese pulso subyacente, y habremos empezado a recuperar para la historia a un número amplio de los verdaderos protagonistas, hoy por hoy en el anonimato absurdo de una historia demasiado obsesiva por lo formal, lo estructurado, y las valoraciones adoptadas con miras aleccionadoras interesadas. 